\title{
Optimization of the Brookhaven ATF Inline-injection System Utilizing PARMELA
}

\author{
Juan C. Gallardo and Harold G. Kirk \\ Physics Department, Bldg. 901-A \\ Brookhaven National Laboratory \\ P. O. Box 5000 \\ Upton, New York 11973-5000
}

\begin{abstract}
An S-band, RF gun-linac, inline-injection system is being installed at the Brookhaven Accelerator Test Facility. An optimization of the system parameters has been done utilizing the electron beam code PARMELA. We describe the results of this procedure and estimate the brightness of the resulting electron beam. We also incorporate the effects of wake fields into the simulation and evaluate their effects on the beam brightness.
\end{abstract}

\section{INTRODUCTION}

The production and maintenance of high-brightness beams has received much attention the last few years. This is due to the realization that such beams are crucial to the success of future linear colliders as well as being very important as radiation sources though such mechanisism as the free-electron laser, the Smith-Purcell effect and Compton scattering. We describe the results of modeling such a beam for the Brookhaven Accelerator Test Facility. For this paper, we define beam brightness to be

$$
B=2 I_{p} / \epsilon_{n}{ }^{2},
$$

where $I_{p}$ is the peak current defined by $Q / \sqrt{2 \pi} \sigma_{\tau}$, with $Q$ and $\sigma_{\tau}$ the electron bunch charge and rms pulse length respectively. $\epsilon_{n}$ is the invariant emittance of the electron beam which we characterize in this paper as the RMS emittance in units of $\pi$ mm-mrad. Clearly, high-brightness beams demand low emittances and, consequently, extensive studies has been made to minimize the emittance of beams at the interaction region. Methods proposed to accomplish this include cooling the beam before its application, reducing emittance growth during the transport of the beam, and minimizing the beam emittance at its production. We report here work toward reducing the beam emittance at the source. These studies have been carried out by modeling the injection system with the $2 \frac{1}{2}$-d simulation code PARMELA ${ }^{1}$. The optimization procedure must incorporate and correlate three main elements of the system: an 


\section{DISCLAIMER}

This report was prepared as an account of work sponsored by an agency of the United States Government. Neither the United States Government nor any agency thereof, nor any of their employees, make any warranty, express or implied, or assumes any legal liability or responsibility for the accuracy, completeness, or usefulness of any information, apparatus, product, or process disclosed, or represents that its use would not infringe privately owned rights. Reference herein to any specific commercial product, process, or service by trade name, trademark, manufacturer, or otherwise does not necessarily constitute or imply its endorsement, recommendation, or favoring by the United States Government or any agency thereof. The views and opinions of authors expressed herein do not necessarily state or reflect those of the United States Government or any agency thereof. 


\section{DISCLAIMER}

Portions of this document may be illegible in electronic image products. Images are produced from the best available original document. 
RF-photocathode gun, a solenoid pair and the linac sections.

A bright beam at the exit of an RF gun does not assure an equivalent beam at a wiggler or final focus. The transport of the beam in conjunction with space-charge forces must be an integral part of the study. A principal cause of emittance dilution in a low-energy section is space-charge forces ${ }^{2,3}$; however, the interplay of space-charge forces with solenoidal forces can lead to a reduction of the transverse emittance inside the linac.

Consider the division of an electron bunch into longitudinal slices of equal length. We follow each slice through the transport system and observe that the intrinsic emittance of each slice is small but that the projection in 2-D phase space of all slices gives rise to the typical fan plot. Although the area swept by each slice is relatively small, the global area covering all slices is significantly larger as is, consequently, the total emittance.

Space-charge forces are greater in the middle core of the beam than at the ends and as a result, the core tends to have larger $\left\langle x^{\prime}\right\rangle$ and $\langle x\rangle$ than the ends which leads to phase space ellipses of different orientations. As a consequence, each slice evolves differently in phase space giving rise to further emittance growth.

As discussed in Ref. 3, it is possible to arrange the focusing and drifts so that the phase space ellipse rotates from the first to the fourth and back again to the first quadrant. Under these conditions, the end sections of the pulse are compressed more than middle sections, leading to stronger space-charge forces in the end sections compared to the middle. For this case, the effects of space charge are reversed from the normal conditions and the fan tends to close, thereby decreasing the total emittance of the bunch. At this point, if the beam is left to drift, space charge will eventually spread the fan again and restore the emittance to its previous higher value. However, in our design, the waist occurs inside of the linac. As the beam is accelerated, the slice ellipses are frozen with respect to each other due to a decrease of space-charge forces which vary as $O\left(\frac{1}{\gamma^{2}}\right)$. The final result after appropriate tuning is an emittance for the entire beam which can approach the individual slice emittances.

This emittance correction scheme only corrects linear forces. Therefore, a uniform laser illumination of the cathode with sharp spatial and temporal boundaries is desirable, as this type of distribution results in linear space-charge forces.

\section{PARMELA RESULTS}

The parameter space for this system is complex. We have chosen to keep some variables fixed while we vary others, in order to study the sensitivity of the solution to the relative stability of the RF and the laser system. As stated above, a uniform beam distribution offers significant advantages over gaussian distributions in terms of minimizing the final beam emittance and is used through out.

While we vary the bunch charge, the radius and bunch length of the initial electron beam and the RF phase; the length of the solenoid was fixed at $15 \mathrm{~cm}$, and the average accelerating gradient of the linac sections was set at $7 \mathrm{MV} / \mathrm{m}$. Parameters used in this analysis are shown in table 1. 


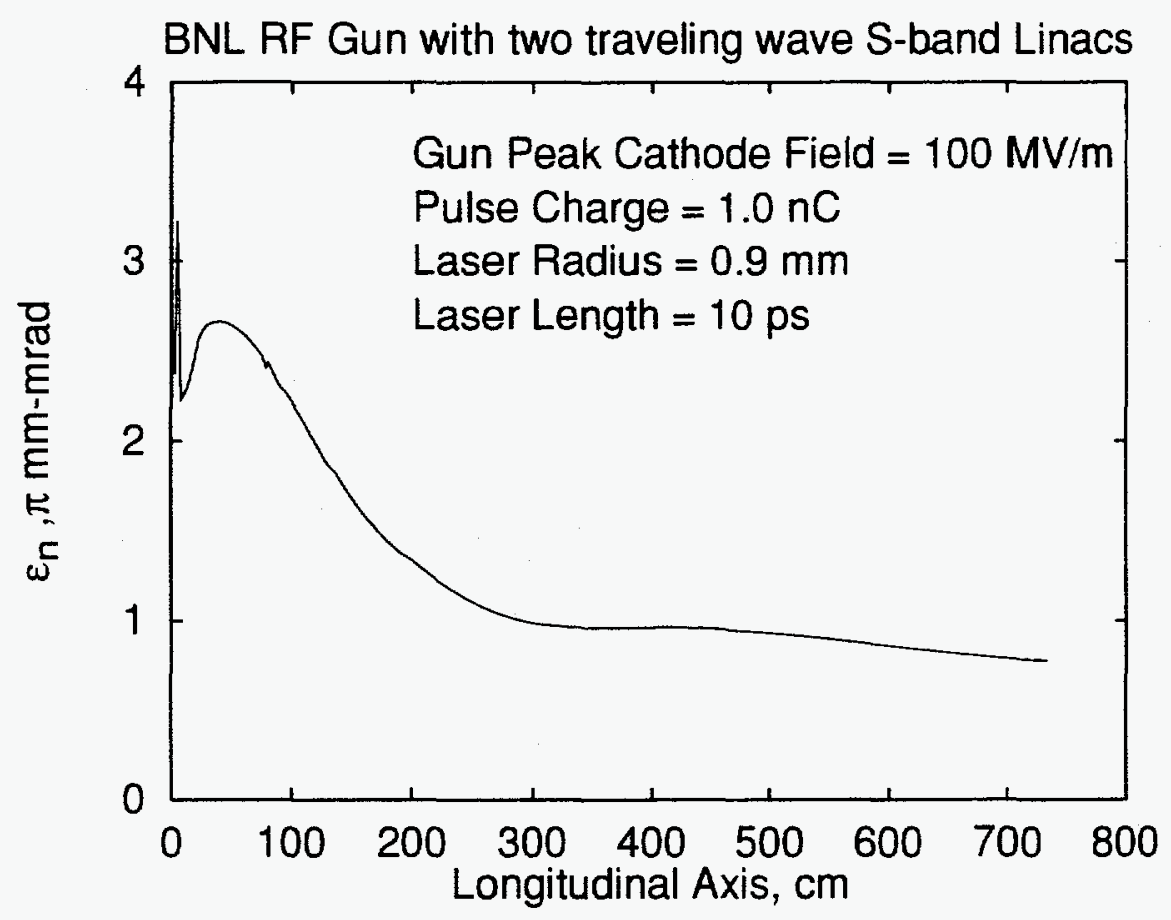

Figure 1: Invariant transverse emittance $\epsilon_{n}$ vs. distance $z$; the plane of the photocathode is $62 \mathrm{~cm}$ from the linac entrance

Table 1. Parameters used in the PARMELA simulations for an axially symmetric $1 \frac{1}{2}$-cell gun in a $\pi$-mode configuration with a solenoid pair.

\begin{tabular}{lc}
\hline \hline RF frequency $[\mathrm{MHz}]$ & 2856 \\
Radius of aperture $[\mathrm{cm}]$ & 1.0 \\
Radius of nose $[\mathrm{cm}]$ & 1.0 \\
First cell length $[\mathrm{cm}]$ & 2.625 \\
Second cell length $[\mathrm{cm}]$ & 5.250 \\
Field on cathode $[\mathrm{MV} / \mathrm{m}]$ & 70 to 200 \\
Initial phase [degree] & 39 to 49 \\
Laser spot size ${ }^{a}[\mathrm{~cm}]$ & 0.05 to 0.24 \\
Laser pulse width ${ }^{a}[\mathrm{ps}]$ & 10 to 14 \\
Charge $[\mathrm{nC}]$ & 1 to 4 \\
Solenoidal field $\mathrm{B}_{0}[\mathrm{kG}]$ & $1.5-3.5$ \\
Solenoid length $[\mathrm{cm}]$ & 15.0 \\
\hline \hline
\end{tabular}

${ }^{a}$ Uniform profile

We show in Fig. 1 a solution for delivering a $1 \mathrm{nC}$ beam through the linac. This result confirms that the emittance compensation technique works when applied to an S-band gun operating with peak axial electric fields at $100 \mathrm{MV} / \mathrm{m}$. The brightness of this beam is $B=5 \times 10^{14} \mathrm{~A} / \mathrm{m}^{2}$.

We explore this effect further by obtaining the minimum transverse emittances achievable when operating the RF gun with different accelerating gradients. The 


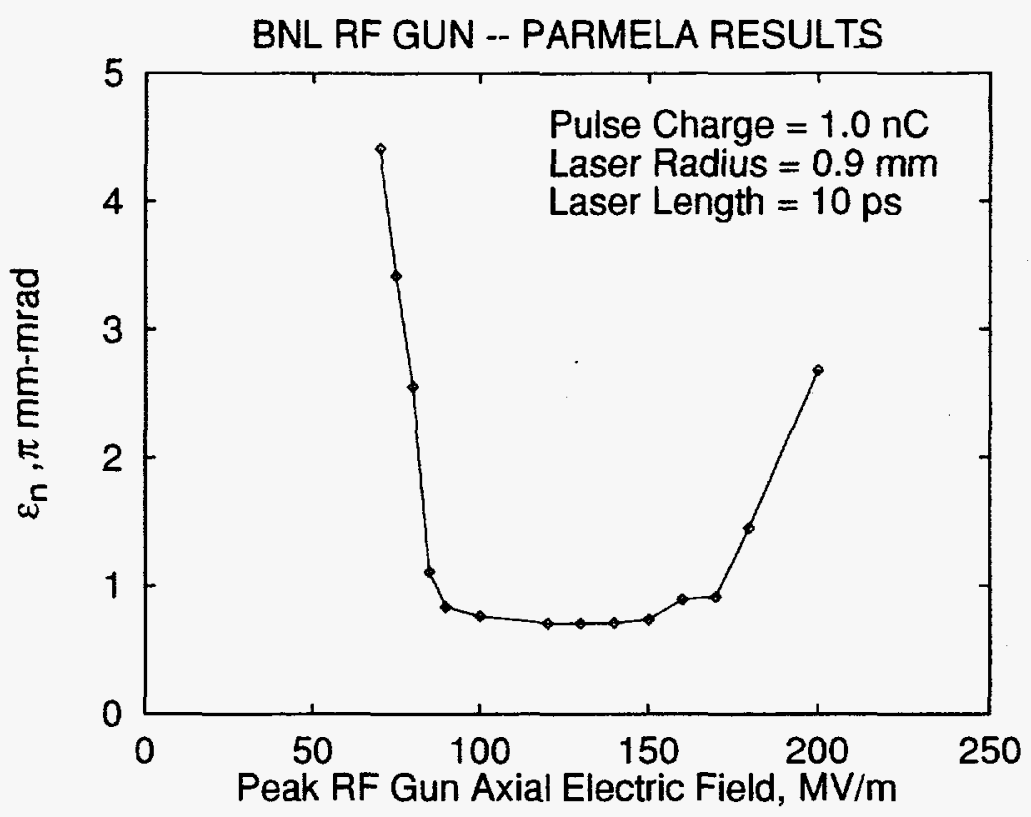

Figure 2: Invariant transverse emittance $\epsilon_{n}$ vs. the cathode peak electric field; the plane of the photocathode is $62 \mathrm{~cm}$ from the linac entrance

results of this analysis is shown in Fig. 2. The strong upturn of emittance at lower accelerating gradients implies that it is advantageous to apply high accelerating gradients at the electron source. These high gradients reduce the development of emittance growth but do not seriously detract from the ability to compensate for the emittance growth that does occur. The upturn in emittance at high accelerating gradients is due to the growing dominance of RF dynamical forces at the gun apertures.

We have further examined the influence of bunch charge on the emittance of the beam emerging from the linac sections. Shown in table 2 are the results obtained when the bunch charges is varied from 1 to $4 \mathrm{nC}$. Note that the brightest beam is obtained for the $1 \mathrm{nC}$ case.

Table 2. Invariant emittance for several bunch charges; the cathode peak field is $100 \mathrm{MV} / \mathrm{m}$

\begin{tabular}{lcccc}
\hline \hline Charge Q [nC] & 1 & 2 & 3 & 4 \\
\hline$\epsilon_{n}[\pi \mathrm{mm}-\mathrm{mrad}]$ & 0.7 & 2.2 & 4.0 & 6.3 \\
Initial phase [ $\left.^{\circ}\right]$ & 49.0 & 47.0 & 44.0 & 39.5 \\
Laser spot size $^{a}[\mathrm{~mm}]$ & 0.9 & 1.4 & 1.9 & 2.4 \\
Laser pulse full width $\left.^{\boldsymbol{a}}{ }^{\circ}{ }^{\circ}\right]$ & 10.0 & 12.0 & 13.0 & 14.0 \\
$\mathrm{~B}_{0}[\mathrm{kG}]$ & 2.14 & 2.15 & 2.12 & 2.11 \\
Final $\sigma_{\boldsymbol{r}}[\mathrm{mm}]$ & 0.5 & 1.0 & 1.5 & 2.4 \\
Final $\sigma_{\boldsymbol{z}}[\mathrm{ps}]$ & 3.38 & 3.98 & 4.00 & 3.97 \\
Final energy spread [\%] & 0.5 & 0.86 & 0.4 & 0.24 \\
\hline \hline
\end{tabular}

${ }^{a}$ Uniform profile 


\section{WAKE-FIELD EFFECTS}

An intense pulse of electrons, traveling down a linear accelerator, interacts with the linac structure and generates electromagnetic fields known as wake fields ${ }^{4,5,6}$. If the beam current is high, the wake fields are strong and have the potential to significantly affect the particle distribution and cause the emittance of the beam to grow. This deterioration of the beam quality will decrease the high brightness of the beam and will compromise the performance of any one of the applications for the electron beam. In particular this effect may impose an important constraint on the shortest wavelength achievable with a free-electron laser, as $\epsilon_{x, y} \leq 2 \pi \lambda$.

Wake fields generated by the head of the beam can be decomposed into several components each producing different dynamical effects on the the tail of the beam: a) the monopole component or longitudinal wake field consists of longitudinal electric fields which are uniform across the cross section of the beam pipe but are a function of the beam longitudinal variable $\eta$. This component give rise to an energy variation or energy spread within the length of the pulse; b) dipole component or transverse wake field which deflects the tail of the bunch in the same direction as the displacement of the head; c) quadrupole component caused by a pulse having a transverse spatial distribution that is not axial symmetric. The later effect is to change the transverse distribution of the tail without affecting the center and head of the pulse even for a properly centered beam.

It is well known that longitudinal wake-field effects can be minimized by adjusting the position of the pulse with respect to the phase of the RF in the linac and that the transverse wake fields can be, in principle, eliminated by proper alignment of the linac and very accurate beam injection. The quadrupole component is much smaller than the other two components and therefore will be ignored; however, we notice that this component cannot be avoided if the beam charge distribution is not cylindrically symmetric.

We have modified the code PARMELA to include the effects of wake fields on the dynamics of the electrons. The new subroutine reads the average wake-field potentials for a structure ${ }^{7}$, computes a spline interpolation and saves the result for later use. As the particles cross the end of a traveling wave cell, wake-field forces are applied according to a simple impulse approximation.

The monopole wake field, $W_{0}\left(\eta_{i}-\eta_{k}\right)$, changes the energy of the particles as

$$
\Delta T_{k}=-L \sum_{i=1}^{k-1} W_{0}\left(\eta_{i}-\eta_{k}\right)
$$

where $\Delta T_{k}$ is the change of kinetic energy of the examined $\mathrm{k}$-th particle, $\mathrm{L}$ is the length of the cell and the sum is over all the particles ahead of the examined $k$-th particle.

The dipole wake field $W_{1}\left(\eta_{i}-\eta_{k}\right)$ causes deflection for a misaligned beam, and is given by

$$
\Delta x_{k}^{\prime}=\frac{r_{e} L}{\gamma_{k}} \sum_{i=1}^{k-1} W_{1}\left(\eta_{i}-\eta_{k}\right) x_{i}
$$




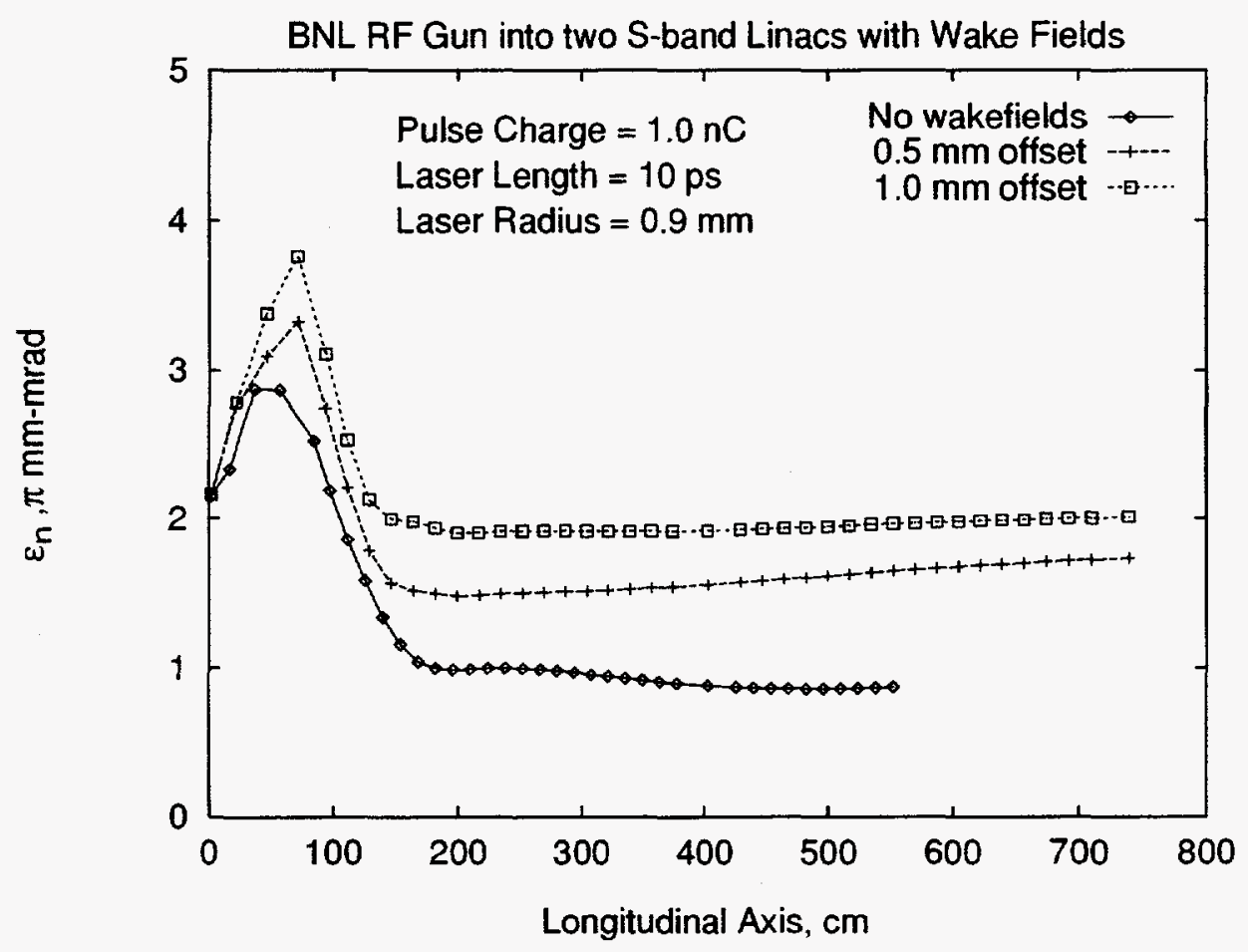

Figure 3: Transverse emittance vs distance $\mathrm{z}$ displaying the effects of wake fields

where the notation is the same as before and $r_{e}$ is the classical electron radius, $\gamma_{k}$ is the normalized energy of the examined $k$-th particle and $x_{i}$ is the transverse displacement of each of the particles ahead of the examined $k$-th particle.

The results of simulations for a $1 \mathrm{nC}$ beam with several displacements are shown in Fig.3. We observe a deterioration of the emittance by nearly a factor of 3 with respect to the unperturbed value for a displacement of $1 \mathrm{~mm}$. Assuming an alignment tolerance of $0.5 \mathrm{~mm}$ we would expect a final emittance $\epsilon_{n} \approx 1.6 \pi \mathrm{mm}$-mrad.

\section{CONCLUSIONS}

We conclude that technique of compensation of emittance growth due to the presence of space-charge forces works well for an S-band $1 \frac{1}{2}$-cell RF gun operating at field accelerating gradients up to $160 \mathrm{MV} / \mathrm{m}$. The preservation of the achieved emittance demands tolerances approaching $0.1 \mathrm{~mm}$ in the subsequent linac sections.

\section{ACKNOWLEDGEMENTS}

The authors wish to thanks D. Yeremian and R. Miller for providing the PARMELA input parameters for the traveling-wave accelerating sections. We also thank $\mathrm{K}$. Bane for providing the optimized average wake field potentials. This research was supported by the U.S. Department of Energy under Contract No. DE-ACO2-76-CH00016. 


\section{REFERENCES}

1. L. Young, private communication.

2. B. Carlsten, New photoelectric injector design for the Los Alamos National Laboratory XUV Fel accelerator, Nucl. Instr. and Meth. A285 (1989) 313; R. L. Sheffield, AIP Conference Proceedings, 184, (1990) 1502 and reference there in.

3. J. Gallardo and H. Kirk, An injection scheme for the Brookhaven ATF utilizing space-charge emittance growth compensation, Proc. of Particle Accelerators Conference (1993) 3615.

4. A. Chao, B. Richter, C.-Y Yao, Nucl. Instr. and Meth., 178 (1980) 1; H. Henke, $C E R N-L E P-R F / 87-36$, Cern preprint (1987).

5. P. Wilson, Physics of High Energy Accelerators, AIP Conf. Proc. No. 87, AIP, New York, (1982).

6. A. Chao, Physics of High Energy Accelerators, AIP Conf. Proc. No. 105, AIP, New York, (1983).

7. K. Bane, Physics of High Energy Accelerators, AIP Conf. Proc. No. 153, AIP, New York, (1987), pp. 971. 